\title{
Review
}

Christian S. Lentz*

\section{What you see is what you get: activity-based probes in single-cell analysis of enzymatic activities}

https://doi.org/10.1515/hsz-2019-0262

Received May 20, 2019; accepted June 25, 2019

\begin{abstract}
Molecular imaging methods can provide spatio-temporal information about the distribution of biomolecules or biological processes, such as certain enzymatic activities, in single cells. Within a cell, it is possible to define the subcellular location of a target, its trafficking through the cell, colocalization with other biomolecules of interest and involvement in certain cell biological processes. On the other hand, single-cell imaging promises to distinguish cells that are phenotypically different from each other. The corresponding cellular diversity comprises the presence of functionally distinct cells in a population ('phenotypic heterogeneity'), as well as dynamic cellular responses to external stimuli ('phenotypic plasticity'), which is highly relevant, e.g. during cell differentiation, activation (of immune cells), or cell death. This review focuses on applications of a certain class of chemical probes, the so-called activity-based probes (ABPs), for visualization of enzymatic activities in the single-cell context. It discusses the structure of ABPs and other chemical probes, exemplary applications of ABPs in single-cell studies in human, mouse and bacterial systems and considerations to be made with regard to data interpretation.
\end{abstract}

Keywords: activity-based protein profiling; chemical probe; enzyme; flow cytometry; molecular imaging; phenotypic heterogeneity.

\footnotetext{
*Corresponding author: Christian S. Lentz, Department of Chemical Biology (CBIO), Helmholtz-Centre for Infection Research, Inhoffenstr. 7, D-38102 Braunschweig, Germany, e-mail: christian.lentz@helmholtz-hzi.de.https://orcid.org/ 0000-0001-7284-2264
}

\section{Introduction: a chemical toolbox to study enzymatic activities at the single-cell level}

Activity-based probes (ABPs) are functionalized active site-directed enzyme inhibitors that traditionally comprise three key elements, a reactive electrophile, a linker (or specificity element) and a functional tag (Figure 1 A,B) (Niphakis and Cravatt, 2014; Sanman and Bogyo, 2014; van Kasteren et al., 2017). The electrophile (also called 'warhead') reacts with the active site nucleophile of a target enzyme family leading to a covalent attachment of the probe to the protein. Different electrophilic groups have been exploited to develop ABPs selective for a variety of enzyme classes, such as serine hydrolases (Liu et al., 1999), cysteine proteases (Kato et al., 2005), glycosidases (Vocadlo and Bertozzi, 2004), etc. As ABPs have a modular structure, different functional tags may be used with the same probe scaffold depending on the application of choice. The most commonly used tags include fluorophores (for detection and imaging), biotin (for enrichment) or functionally flexible click-chemistry handles for bio-orthogonal attachment of other tags in situ. ABPs may have different selectivity profiles ranging from single-target specificity to broad-spectrum activity toward all members of one or more enzyme families. Target selectivity is dictated by the combination of structural components of the probe and the resulting steric demand and electrophilicity. While for some ABPs, linker and warhead may not be completely distinguished, particularly in protease-directed ABPs the linker region connecting the warhead and reporter tag is usually well defined and highly important for probe selectivity (see Chakrabarty et al., 2019, for a recent review on ABPs targeting proteases). As proteases recognize physiological substrates through certain peptidic sequence motifs, these cleavage preferences can be elucidated through so-called substrate profiling methods, 
A

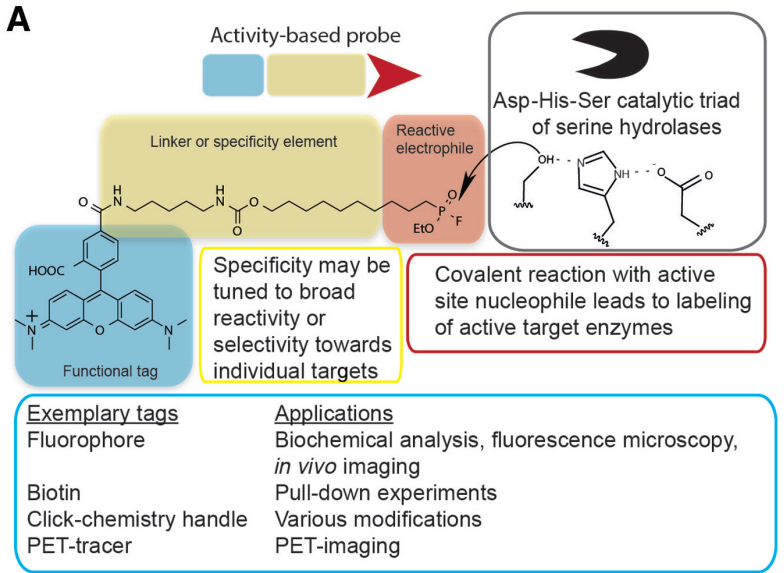

C

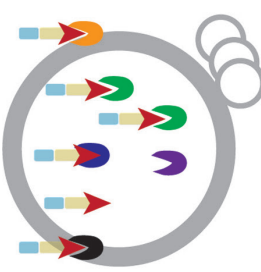

Condition 1

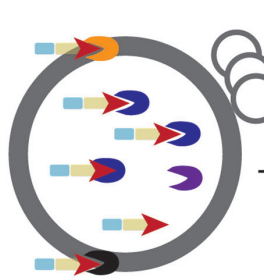

Condition 2

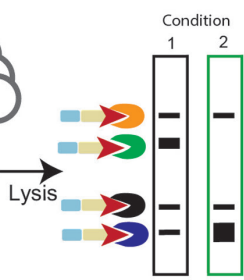

SDS-PAGE fluorescence scan
B

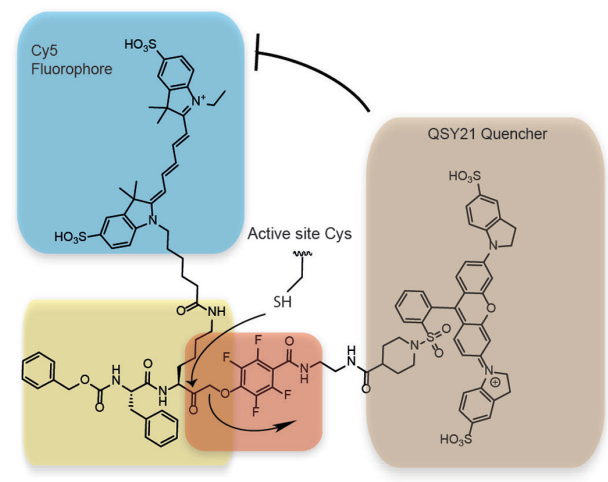

\section{D}



Flow cytometry

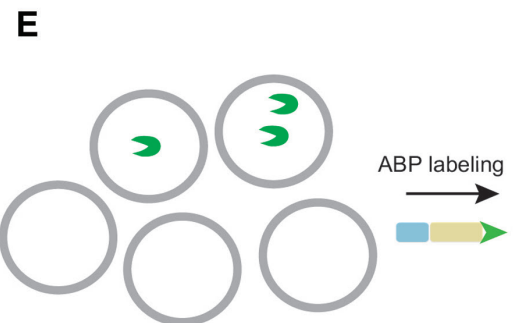

Heterogenous cell population

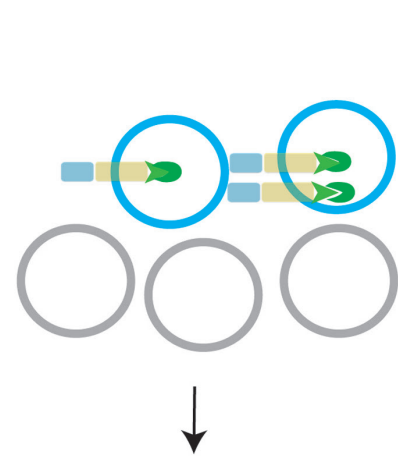

Single-cell proteomic analysis

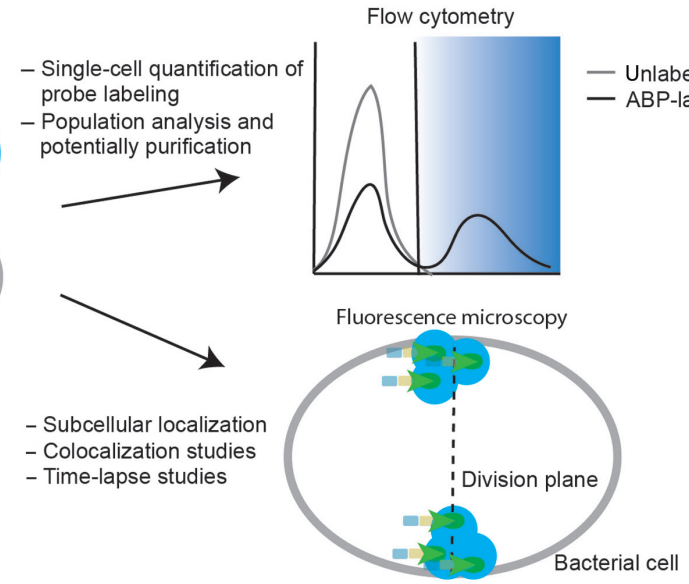

Figure 1: Introduction into ABP structures and their application in activity-based protein profiling and single-cell analysis. (A) General structure of ABPs illustrated using the structure of the general $\alpha, \beta$-hydrolase probe FP-TMR (Liu et al., 1999). (B) Structure of the quenched ABP BMV109 (Verdoes et al., 2013) used to visualize cysteine cathepsin activity. (C) Schematic representation of activity-based protein profiling using fluorescent ABPs and (D) a competitive ABPP approach to identify selective inhibitors of individual targets.

(E) Illustration of single-cell approaches using a target-selective $A B P$.

e.g. through fluorogenic substrate libraries (Harris et al., 2000; Kasperkiewicz et al., 2014) or mass-spectrometrybased peptidic library screening methods (O’Donoghue et al., 2012). These structural determinants of protease cleavage specificity may then be harnessed to develop peptidic specificity elements that render the probe target-selective (Kasperkiewicz et al., 2014, 2015; Poreba et al., 2014; Lentz et al., 2016).
Activity-based protein profiling (ABPP) is the most widespread application of ABPs, for which usually broadly reactive ABPs are used to globally detect and resolve all active members of a group of enzymes in a biological sample (Figure 1C) (Liu et al., 1999; Kato et al., 2005). Targets of fluorescent ABPs can be analyzed biochemically by sodium dodecyl sulfate polyacrylamide gel electrophoresis (SDS-PAGE) analysis, whereas biotinylated or 
'clickable' probes can be used for enrichment of target proteins for identification by mass spectrometry. This strategy can also be applied to identify the molecular targets of natural products (reviewed in Wright and Sieber, 2016; Nomura and Maimone, 2019) or off-targets of chemotherapeutic drugs (van Esbroeck et al., 2017). The use of ABPP in a variety of different biological areas has been the subject of a number of reviews, e.g. for cancer (Nomura et al., 2010), plant biology (Morimoto and van der Hoorn, 2016), microbiology (Patel et al., 2019; Sharifzadeh et al., 2019; Whidbey and Wright, 2019), the host-pathogen interface (Kovalyova and Hatzios, 2019), host-virus interactions (Desrochers and Pezacki, 2019) or immunology (Borne et al., 2019; Koenders et al., 2019).

In drug discovery, ABPP is often performed in a competitive format in which chemical libraries are screened in order to identify inhibitors that compete with ABP labeling of single targets, allowing for a simultaneous assessment of the selectivity profile of the compound against other members of the target enzyme family (Figure 1D) (Niphakis and Cravatt, 2014). In addition to these chemoproteomic approaches, fluorescent ABPs can be used for molecular imaging of enzyme activities in systems ranging from in vitro cellular models up to non-invasive in vivo imaging in animals and emerging clinical diagnostic applications (Blum et al., 2005, 2007; Edgington et al., 2009; Verdoes et al., 2012, 2013; Garland et al., 2016).

Other types of chemical tools, such as substrates or metabolic labeling strategies, may also be suitable to visualize enzymatic activities in cells. Although the detailed discussion of these strategies is beyond the scope of this review, they shall be briefly introduced and a few outstanding examples will be discussed later. In contrast to ABPs, substrate probes must be enzymatically cleaved by their target in order to generate a detectable fluorescent signal. Quenched fluorescent substrates feature a fluorophore and quencher on opposing ends of the molecule and enzymatic cleavage leads to the generation of a fluorescent signal (Ofori et al., 2015). Alternatively, such probes may possess a caged fluorophore or fluorogenic group that is activated upon enzymatic processing, or they may be based on fluorescence-resonance energy transfer (FRET) between two fluorophores (as reviewed in Hu et al., 2014). Due to the continuous turnover of multiple copies of substrates per target enzyme, these probes easily yield higher fluorescent signals compared to ABPs which bind their target with a 1:1 stoichiometry. Yet, this increase in signal comes with a loss of spatial resolution as the fluorescent reporter will diffuse away from the site where it has been generated. As an emerging, highly sensitive alternative to fluorophores in chemical imaging probes, chemiluminescent leaving groups are emerging, as reported by Shabat and coworkers who developed an adamantylidene-dioxetane-based probe with an analyteresponsive (e.g. protease-cleavable) protecting group (Roth-Konforti et al., 2017).

Another strategy for biomolecular imaging that exploits the enzymatic machinery of cells is metabolic labeling, i.e. the incorporation of functionalized analogs of biomolecules into cellular structures. This strategy has found particular relevance in prokaryotic cell biology where fluorescently labeled precursors (or precursors equipped with a bio-orthogonal handle allowing attachment of a fluorophore vial click-chemistry at a later step) of the cell envelope can be used to probe the mechanisms of cell wall biogenesis or the principles of bacterial cell division and growth (as reviewed in Siegrist et al., 2015).

The use of ABPs for single-cell analysis is particularly beneficial to capture dynamic changes in cellular phenotypes by molecular imaging and can be used as molecular markers for functionally distinct cellular states in heterogenous cell populations (Figure 1E). In the following, we will therefore discuss exemplary applications of different types of ABPs for single-cell analysis in a broad range of different biological settings from proteases in immune cells, the tumor microenvironment and cell death to the use of antibiotic-based ABPs to study microbial cell biology.

\section{Cysteine cathepsins and macrophage activation - a short history of molecular imaging with ABPs}

By far the largest body of literature on molecular imaging using ABPs focuses on cysteine cathepsins (Cat), a group of 11 lysosomal papain-like cysteine proteases (review in Turk et al., 2012). In addition to their involvement in terminal lysosomal protein degradation, cysteine cathepsins exert a variety of distinct physiological functions, e.g. in antigen presentation or bone remodeling, and have pathophysiological roles in cancer and a variety of other disorders (Turk et al., 2012). The technological advances in cathepsin $\mathrm{ABP}$ as well as their expanding range of biological applications can therefore be regarded as a paradigm example for the evolution of the ABP-imaging field. The first ABPs shown to target cysteine cathepsins were analogs of the epoxide-based general cysteine protease inhibitor E-64 (Joyce et al., 2004). The use of 
A

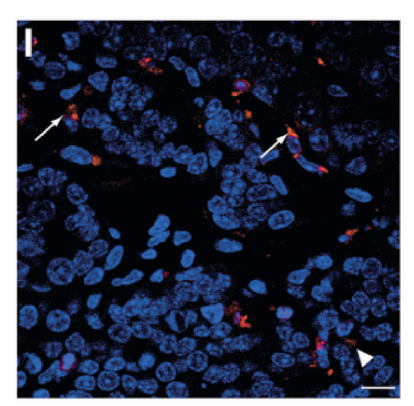

B

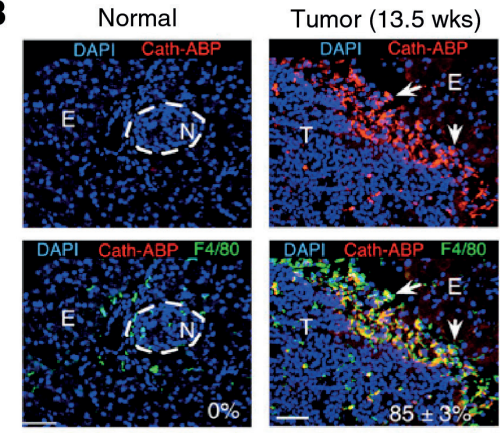

C

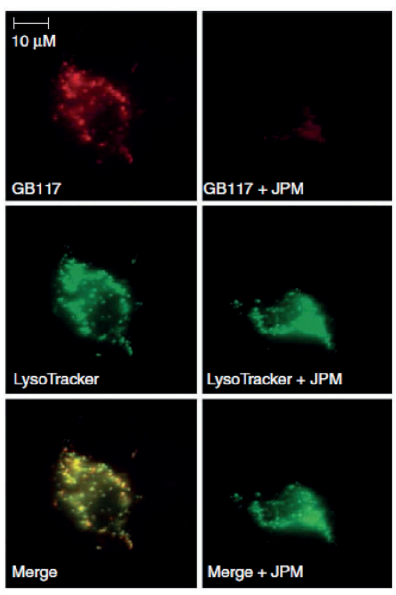

E

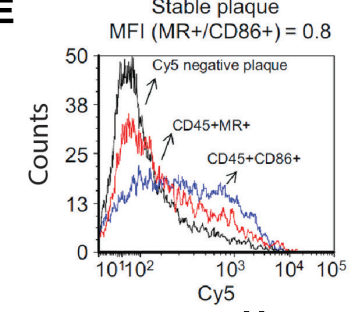

D

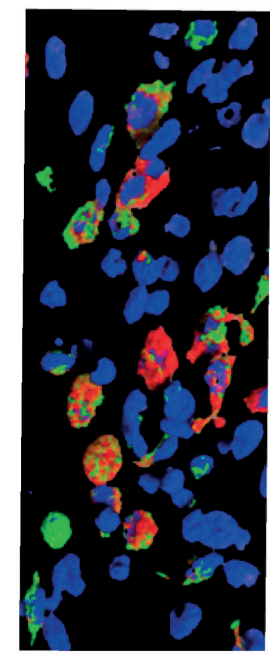

Unstable plaque

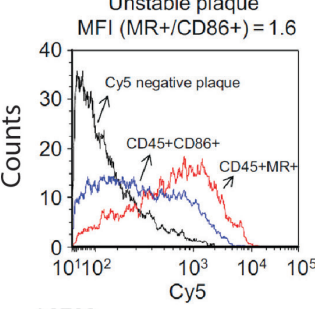

$\mathbf{F}$

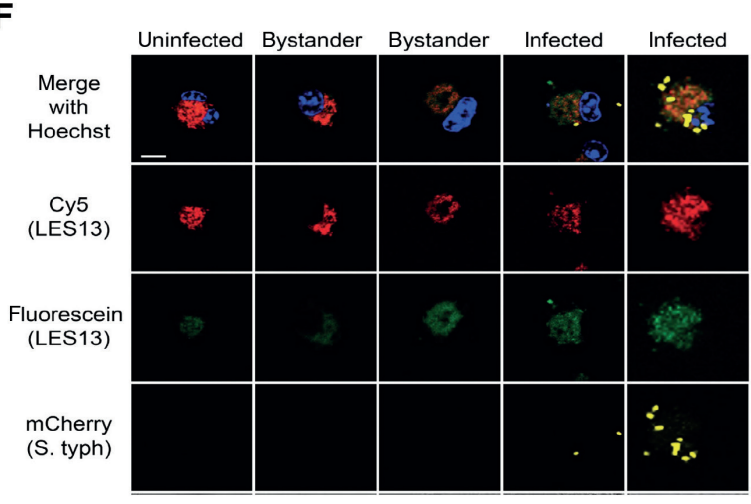

$\mathbf{H}$

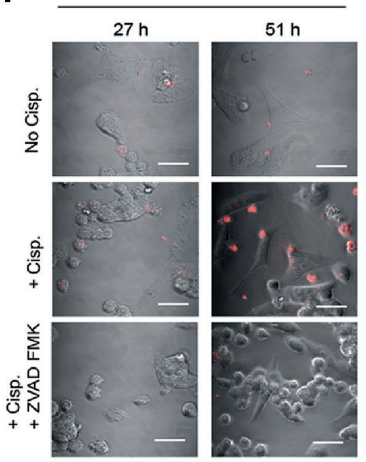

G

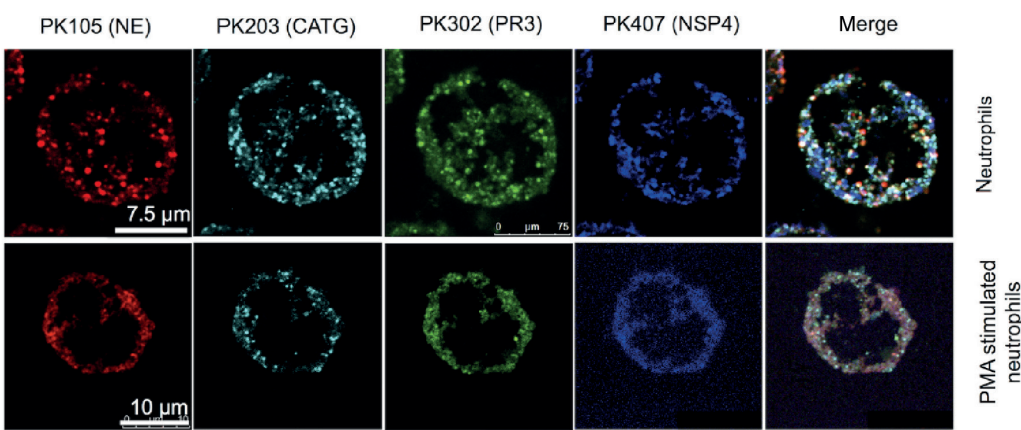

a biotinylated probe (DCG-04) revealed a progressive increase in cathepsin activity during tumorigenesis in a murine model of pancreatic islet cell carcinogenesis (RIP1Tag2 mice) as assessed by SDS-PAGE analysis (Joyce et al., 2004). A fluorescent derivative (BODIPY530/550-DCG04) was then applied in vivo to Rip1-Tag2 mice, revealing distinct cellular localization patterns of cathepsin activity during RIP-Tag tumorigenesis by confocal microscopy of tissue slides (Figure 2A) (Joyce et al., 2004). Separation of the constituent cell types by flow cytometry followed by DCG-04 labeling of the sorted cell types revealed that each cell type had a characteristic profile of different cathepsin activities with infiltrating immune cells showing the highest levels of activity (Joyce et al., 2004). A follow-up 
Figure 2: Examples of single-cell analysis of human or mouse cells with ABPs.

(A) Tumor tissue slides from Rip1-Tag2 mouse treated intravenously with a fluorescent derivative of cysteine-protease probe DCG04 (red) reveal cathepsin activity in a subset of cells [blue: 4',6-diamidino-2-phenylindole (DAPI)]. Scale bar: $10 \mu \mathrm{m}$. The figure is reprinted with permission from Joyce et al. (2004). (B) Pancreatic tissue slides from normal and RT2 tumor-bearing from mice treated with a fluorescent cathepsin-ABP (Cath-ABP, red) were counterstained with an anti-F4/80 antibody (green) and DAPI (blue). The numbers indicated in each graph show the percentage of Cath- $\mathrm{ABP}^{+}$cells in the $\mathrm{F} 4 / 80^{+}$population. The figure is adapted with permission from Gocheva et al. (2010). (C) Specific lysosomal labeling of cathepsins with a quenched fluorescent cathepsin-ABP (GB117). Fluorescence microscopy images of NIH-3T3 cells labeled with the GB117 (red) after preincubation with vehicle control or the cysteine protease inhibitor JPM-OEt. Lysosomal compartments were counterstained with LysoTracker (green). The figure is reprinted with permission from Blum et al. (2005). (D) Cathepsin activity in the tumor microenvironment localizes to a subset of $\mathrm{CD} 68^{+}$macrophages. Three-dimensional reconstruction of confocal fluorescence microscopy images taken from tumor of mice treated with the quenched-fluorescent cathepsin ABP BMV109 (red). Tissues were co-stained with anti-CD68 immunostaining and DAPI (blue). The figure is reprinted with permission from Verdoes et al. (2013) Copyright (2013) American Chemical Society. (E) Histograms showing the distribution of Cy5-fluorescence (resulting from labeling with the cathepsin probe GB123) in $\mathrm{CD} 45^{+} \mathrm{CD} 86^{+}$and $\mathrm{CD} 45^{+} \mathrm{MR} \mathrm{R}^{+}$macrophages from stable or unstable atherosclerotic plaques as determined by flow cytometry. MR: mannose receptor CD206. The figure is reprinted with permission from Abd-Elrahman et al. (2016). (F) Confocal microscopy images of bone marrow-derived monocytes infected with mCherryexpressing S. typhimurium (yellow) and labeled with the bifunctional Cat-pH-probe LES13. Cathepsin-activity-dependent Cy5 fluorescence is pseudocolored in red, and pH-dependent fluorescein fluorescence in green. For bystander cells two representative images are shown reflecting cells with lower and higher $\mathrm{pH}$. For infected cells two representative images of cells with different numbers of intracellular bacteria are shown. The figure is reprinted with permission from Sanman et al. (2016b). Scale bar: $20 \mu \mathrm{m}$. (G) Human neutrophils [freshly isolated or after phorbol myristate acetate (PMA) stimulation] labeled with selective activity-based probes for neutrophil elastase (PK105, red), cathepsin G (PK203, cyan), proteinase 3 (PK302, green) and neutrophil serine protease 4 (PK407, blue) revealing heterogenous granular distribution of serine proteases. The figure is reprinted with permission from Kasperkiewicz et al. (2017) Copyright (2017) American Chemical Society. (H) Timecourse of cisplatin-induced apoptosis in A2780 cells as visualized by labeling with the caspase 3-selective ABP AB46-Cy5 (red) with or without pretreatment with the caspase-inhibitor Z-VAD-fmk. The figure is reprinted with permission from Shaulov-Rotem et al. (2016).

study demonstrated that $\mathrm{F} 4 / 80^{+}$macrophages present in early stages of tumorigenesis did not stain with a cathepsin probe, but that in the course of RIP1-Tag2 tumorigenesis a subset of $\mathrm{F} 4 / 80^{+}$macrophages emerged as the major cellular targets for cathepsin (CatB, CatS) probe labeling (Figure 2B) (Gocheva et al., 2010). This finding is consistent with the observation that tumor-associated macrophages have $\sim 12$-fold elevated cathepsin activity levels compared to blood monocytes and suggests that the probe labels a subpopulation of macrophages specifically activated in the tumor microenvironment (Gocheva et al., 2010). The authors continued to demonstrate that interleukin (IL)-4, which in the tumor microenvironment is produced at high levels by $\mathrm{T}$ cells and tumor cells and polarizes macrophages to an alternatively activated M2-type state, induces cathepsin activity in macrophages (Gocheva et al., 2010).

Subsequent advances in cathepsin probes have focused on two shortcomings of the DCG-04 probe that limit its utility for imaging studies: The broad reactivity profile prevents any conclusion about the specific member of the cathepsin family contributing to the fluorescence signal and hence their specific biological function. The development of ABPs with selectivity for individual cathepsins will be discussed in the next section of this review. Second, high background due to non-specific retention of free fluorescent probe may potentially limit signal-tonoise contrast for live imaging applications that do not allow washing to separate free from target-bound probe.
The latter problem was addressed by the development of quenched fluorescent activity-based probes (qABPs) based on an acyloxymethylketone (AOMK) warhead that become fluorescent only after activity-based covalent modification of the target protein (Blum et al., 2005). In the probe GB117, a QSY7 quencher is incorporated into the leaving group of the AOMK warhead and a BODIPYtetramethylrhodamine (TMR)-X fluorophore is conjugated to the $\varepsilon$-amino group of the $\mathrm{P}_{1}$ lysine residue. (To describe protease specificity a positional nomenclature is used where $\mathrm{P}_{1}$ denotes the position of the first amino acid $\mathrm{N}$-terminal of the cleavage site, the second amino acid is denoted as $\mathrm{P}_{2}$, the third as $\mathrm{P}_{3}$ and so forth. Amino acids' C-terminal of the cleavage site are denoted as $\mathrm{P}_{1}^{\prime}$, etc...). When used for live imaging in $\mathrm{NIH}-3 \mathrm{~T} 3$ cells, the quenched probe GB117 exclusively labeled lysosomal compartments (which harbor cathepsins) and colocalized with the lysosomal marker Lysotracker (Figure 2C) (Blum et al., 2005). In contrast, the non-quenched derivative GB111 showed non-specific staining of the entire cell under the same conditions, although the non-lysosomal labeling could be washed out by prolonged incubation in probe-free medium (Blum et al., 2005). When applied to three-dimensional spheroids of the fibrocystic breast-cell line MCF-10A, probe GB117 was found to predominantly label lysosomal compartments at apical poles of the cells, whereas immunostaining with an antibody against Cat B (the main target of GB117 besides Cat L) labeled both basal and apical poles of acinar structures (Blum et al., 2005). 
The authors attributed these differences in subcellular labeling patterns to discrepancies between the labeling of total protein (as visualized by antibody staining) and active protein (as visualized by the ABP) (Blum et al., 2005).

The next generation of cathepsin probes was optimized for non-invasive in vivo imaging applications. Due to the increased tissue penetration of near-infrared light, the use of a near-infrared fluorophore quencher pair (Cy5/ QSY21) in probe GB137 allowed the detection of tumors by non-invasive in vivo imaging of mice based on the increased levels of cathepsin activity in the tumor microenvironment (Blum et al., 2007). This probe was further optimized by replacing the AOMK warhead with a more reactive 2,3,5,6-tetrafluoro-substituted phenoxymethylketone electrophile (PMK) (Verdoes et al., 2013). The resulting probe BMV109 (Figure 1B) displayed activity toward cathepsins and possessed highly improved imaging properties (Verdoes et al., 2013). This study also included confocal microscopy of BMV109-treated tumor tissue that was immunohistochemically counterstained for the macrophage marker CD68 (Verdoes et al., 2013). Intriguingly, only a subset of $\mathrm{CD}^{+} 8^{+}$-macrophages stained with the cathepsin probe suggesting the presence of distinct macrophage subpopulations with a different activation state (Figure 2D) (Verdoes et al., 2013).

Abd-Elrahman et al. employed a fluorescent ABP (GB123, the unquenched analogs of GB137) to visualize increased levels of cathepsin (CatB, CatS) in unstable (i.e. high risk) over stable (low risk) atherosclerotic plaques (Figure 2E). Microscopic analysis revealed that GB137 colocalized with $\mathrm{CD}^{+} 8^{+}$macrophages and flow cytometry was used to further analyze whether the increased cathepsin activity in unstable plaques could be attributed to $\mathrm{CD} 86^{+}$ classically activated (M1 type) or mannnose receptor (MR) ${ }^{+}$ alternatively activated (M2 type) macrophages. While there was a slight increase in the percentage of $\mathrm{MR}^{+}$cells among the $\mathrm{CD} 45^{+}$monocyte population in unstable compared to stable plaques, the percentage of $\mathrm{GB}_{12} 3^{+}$cells in the $\mathrm{MR}^{+}$ population in unstable plaques was increased by 5 -fold. The authors therefore suggest the existence of a specific subpopulation among $\mathrm{MR}^{+}$(M2 type) macrophages that may be responsible for the observed increase in cathepsin activities in unstable plaques. This study highlights the utility of ABPs as markers of functional diversity among macrophages beyond the M1 and M2 classification.

A great example for the use of chemical probes to study different fates of individual cells and even individual organelles within cellular subpopulations is a study by Sanman et al. who developed bifunctional cathepsin probes. These probes were built on the Cy5-based quenched cathepsin-ABP BMV109 and were additionally functionalized with a dye that elicits $\mathrm{pH}$-dependent fluorescence to simultaneously study the $\mathrm{pH}$ of the endolysosomal vacuoles where cathepsin activity is found (Sanman et al., 2016b). As the intracellular bacterial pathogen Salmonella typhimurium resides in specialized vacuoles in macrophages, the authors used the bifunctional Cat-pH-probe to analyze the interplay of $\mathrm{pH}$, cathepsin activity and bacteria in vacuolar compartments over the course of S. typhimurium macrophage infection (Figure 2F) (Sanman et al., 2016b). At early infection time points, cathepsin activity could be detected in the same vacuolar compartments as fluorescently labeled bacteria, but organelles containing cathepsin activity in infected cells had elevated $\mathrm{pH}$ compared to the corresponding organelles in bystander cells (Sanman et al., 2016b). In contrast, at $14 \mathrm{~h}$ post infection, when bacteria have established their replicative niche, vacuoles harboring bacteria excluded cathepsin activity and infected cells showed overall lower levels of cathepsin activity (Sanman et al., 2016b). Furthermore, organelles with cathepsin activity in infected cells and some organelles in bystander cells had elevated $\mathrm{pH}$ compared to organelles in cells from non-infected control samples (Sanman et al., 2016b).

\section{Target-specific probes for lysosomal cysteine proteases}

The broad-spectrum activity of cathepsin probes mentioned so far makes them useful to distinguish different cell types and even macrophage subpopulations based on their levels of total cathepsin activity. The deconvolution of the corresponding molecular targets (i.e. the specific combination of targeted cathepsins) of these probes, however, can only be achieved biochemically at the population level. In order to provide single-cell information on the activity of individual members of the cysteine cathepsin family, one must develop target-selective probes.

Patterson et al. reported the identification of cathepsin S-specific 1,4-disubstituted-1,2,3-triazole-based aldehyde inhibitor by a fragment-based screening method for protease inhibitors termed substrate activity screening (Patterson et al., 2006). In order to design a CatS-specific probe, this structural information was used to optimize the scaffold of the AOMK-based general cathepsin probe GB137 yielding BMV083 as a CatS-selective qABP (Verdoes et al., 2012). Flow cytometry analysis of single-cell suspensions generated from mouse 4T1 syngeneic breast tumors showed that BMV083 labeling (and hence CatS activity) 
is highest in tumor-promoting $\mathrm{CD} 45^{+} \mathrm{F} 4 / 80^{\mathrm{HI}} \mathrm{MR}^{+} \mathrm{M} 2$-type macrophages (Verdoes et al., 2012).

Poreba et al. used Hybrid Combinatorial Substrate Library (HyCoSuL) profiling information (which is explained in more detail in the following section) to generate a tetrapeptide-AOMK-based fluorescent probe for CatL (Poreba et al., 2018). Quantitative pixel-by-pixel analysis of multiple fluorescent microscopy images of MDA-MB-231 cells stained by the CatL-ABP showed colocalization with immunostaining by an $\alpha$-CatL antibody and only a partial overlap with $\alpha$-CatB antibody staining, demonstrating the selectivity of the CatL probe (Poreba et al., 2018).

Legumain, also known as asparaginyl endopeptidase, is another lysosomal papain-like cysteine protease involved in major histocompatibility complex (MHC) II antigen processing and is linked to inflammatory disorders and cancer. A quenched fluorescent legumain-specific probe strongly labeled $\mathrm{CD} 68^{+}$macrophages in tissues from patients with chronic pancreatitis (Edgington-Mitchell et al., 2016). While legumain inhibition did not attenuate the progression of pancreatitis in a mouse model, the authors suggested its utility as a potential biomarker (Edgington-Mitchell et al., 2016).

\section{Imaging of neutrophil proteases}

Drag and co-workers have designed a suite of specific fluorescent ABPs to study the granular serine proteases of human neutrophils (Kasperkiewicz et al., 2014, 2015, 2017). In order to achieve the necessary target specificity of probes, they established a methodology termed HyCoSuL profiling that exploits the structural diversity and enlarged chemical space provided by non-canonical amino acids (Kasperkiewicz et al., 2014). The first step of the probe design strategy involves screening of HyCoSuL fluorogenic substrate libraries to determine the natural and non-canonical amino acids preferentially recognized by the target of interest in the four positions of the $\mathrm{N}$-terminal of the proteolytic cleavage site (Kasperkiewicz et al., 2014). These amino acid determinants of substrate specificity can then be combined to generate optimized (tetra)peptidic recognition elements for target-selective fluorogenic (e.g. coumarin based) substrates or ABPs that are functionalized with a detection tag at the $\mathrm{N}$-terminus of the peptidic recognition element and a C-terminal reactive electrophile (e.g. diphenylphosphonate) allowing for covalent target modification (Kasperkiewicz et al., 2014). In a first report on this technology, the biotinylated ABP PK101 was developed with selectivity for neutrophil elastase (NE) (Kasperkiewicz et al., 2014). Imaging with this probe using a fluorescent streptavidin conjugate in a two-step labeling process revealed that $\mathrm{NE}$ activity was restricted to granular locations inside neutrophils and that active protein could hardly be detected in neutrophil extracellular traps (NETs) (Kasperkiewicz et al., 2014). Kasperkiewicz and coworkers continued to design specific fluorescent ABPs for the four major serine proteases in human neutrophils, i.e. NE, cathepsin G, proteinase 3 and neutrophil proteinase 4 (Kasperkiewicz et al., 2017). In parallel imaging studies using ABPs equipped with different orthogonal fluorophores (and confirmed through the use of fluorescent antibody conjugates for the different serine proteases), the authors demonstrated that the four proteases reside in distinct granule subpopulations and do not commonly colocalize with one another (Figure 2G, Kasperkiewicz et al., 2017). This result raises the question about how these distinct granules are populated in neutrophil progenitor cells, and the authors suggest distinct sorting signals in the C-terminal peptides or kinetically or spatially controlled translation of the different serine proteases (Kasperkiewicz et al., 2017). Another open question is whether neutrophils are able to mount fine-tuned functional responses resulting in the coordinated release of only certain subtypes of granules.

Liu et al. recently developed a non-peptidic fluorogenic probe (NEP) for NE activity based on the nearinfrared hemicyanine dye (Liu et al., 2019). When used for fluorescence-microscopy imaging in cell culture, the probe enabled monitoring of subcellular localization and trafficking of NE in adherent leukemia RBL-2H3 cells as well as uptake of exogenous NE into A549 cells. The authors observed a marked increase in cellular NE labeling upon stimulation of RBL-2H3 cells with pro-inflammatory factors such as lipopolysaccharide (LPS), IL-6 and tumor necrosis factor (TNF)- $\alpha$ (Liu et al., 2019) demonstrating that the probe is able to capture dynamic differences in neutrophil activity states. Although not commented on by the authors, the cellular probe-labeling patterns shown in fluorescence-microscopy images suggest a heterogenous response of the cell populations to these stimuli (Liu et al., 2019).

Schultz and coworkers developed a cathepsin G-specific FRET-based peptidic substrate (mSAM) that was targeted to the cell membrane with a palmitate anchor to report on the activity of cathepsin $\mathrm{G}$ at the cell surface upon secretion (Guerra et al., 2019). The authors demonstrated that mSAM can distinguish neutrophils of different activation states both microscopically and by flow cytometry (Guerra et al., 2019). As the probe was activated by neutrophils in sputa from cystic fibrosis patients, but 
not by neutrophils from healthy individuals, the authors suggest its use as a cellular marker to detect airway inflammation (Guerra et al., 2019).

\section{Visualizing cell death pathways with caspase probes}

Another dynamic cellular process characterized by the activation of proteolytic markers is cell death. Apoptosis is dependent on a cascade of proteolytic events executed by caspases, a group of cysteine proteases that cleave their substrates after an aspartic acid residue. In 2000 Bedner et al. reported the use of fluorescent ABPs (before the term $\mathrm{ABP}$ was coined) based on a peptidic caspase recognition motif (VAD for general caspases, VEID as a caspase-6-specific signal) in combination with a C-terminal fluoromethyl ketone ( $f m k$ ) electrophile (for covalent modification of the active site cysteine) and an $\mathrm{N}$-terminal fluorescent tag (carboxyfluorescein, fam) (Bedner et al., 2000). Application of these ABPs (that were named fluorochrome-labeled inhibitors of caspases, FLICA) to human breast carcinoma MCF-7 cells treated with a DNA topoisomerase I inhibitor to induce apoptosis, revealed that a subset of cells became fluorescent (Bedner et al., 2000). As expected, probelabeled cells also showed morphological signs characteristic of apoptosis, such as shrinkage and blebbing (Bedner et al., 2000). The authors also noted a marked degree of heterogeneity in labeling of individual cells by the famVAD-fmk probe and hypothesized that cells vary in their composition of active caspases. In contrast to this pancathepsin probe, the putatively caspase- 6 selective probe fam-VEID-fmk labeled a smaller subpopulation of cells (Bedner et al., 2000). Although the authors confirmed that labeling by the probe correlated with the presence of DNA strand breaks detected by terminal deoxynucleotidyl transferase dUTP nick end labeling (TUNEL) assay (Bedner et al., 2000), it should be noted that these studies did not include control probes unable to bind to caspases either by modifying the peptidic recognition element or by removing the electrophilic warhead required for covalent modification of the active site cysteine. Later it was reported that fmk-based FLICA probes are also able to interact with cysteine cathepsins (Pozarowski et al., 2003). Although a number of fluorescent and even quenched fluorescent caspase-directed ABPs with improved, narrower selectivity profiles had been generated since then (Berger et al., 2004; Edgington et al., 2009, 2012), the first truly selective ABP has been developed recently for caspase-3 by Shaulov-Rotem et al. (Figure 2H) (Shaulov-Rotem et al., 2016).
In a stepwise process, the authors first synthesized a small library of AOMK-based inhibitors and screened their activity against caspase 3 and legumain, the main off-target of many previous AOMK-based caspase probes. The most potent and selective candidates were then converted into fluorescent probes and finally into qABPs based on the Cy5/QSY21 fluorophore/quencher pair (Shaulov-Rotem et al., 2016). A quenched probe was used to dynamically image the progression of apoptosis in MM1 cells upon treatment with etoposide (Shaulov-Rotem et al., 2016) and it also provided new insight into the subcellular location of the target enzyme in apoptotic adherent ovarian carcinoma cells A2780 upon treatment with cis-platin (Shaulov-Rotem et al., 2016). Live-cell imaging in combination with different organelle-specific markers detected caspase- 3 activity in the cytosol, mitochondria and the endoplasmic reticulum (ER) (Shaulov-Rotem et al., 2016). The unexpected localization of caspase-3 activity in the ER was confirmed immunohistochemically using cleaved caspase-3-specific antibodies as well as by fractionation experiments with probe-labeled cells and identification of the probe target by mass spectrometry (Shaulov-Rotem et al., 2016). As A2780cis cells, which are resistant to cisplatin-induced apoptosis, show a faster and more pronounced localization of caspase- 3 to the ER, the authors hypothesized that this localization may bring caspase-3 in the proximity to apoptosis inhibitors in the ER effectively limiting the progress of apoptosis (Shaulov-Rotem et al., 2016).

Not being part of the proteolytic cascade in apoptosis, caspase- 1 is part of the NOD-like receptor family and pyrin domain containing 3-inflammasome that is activated through the engagement of pattern-recognition receptors by pathogen- or other danger-associated molecular patterns (Man et al., 2017). Upon recruitment to adaptor proteins of the inflammasome, the zymogen pro-caspase-1 then activates itself by autoproteolysis before being able to perform several proteolytic tasks generating inflammatory signals, such as the processing of pro-Interleukin (IL)-1B to IL-1 $\beta$, in a process triggering a specific form of programmed cell death known as pyroptosis (Man et al., 2017). Puri et al. synthesized AWP28, a fluorescent AOMKprobe with a caspase-1 optimized tetrapeptide element, which predominantly labeled caspase-1, but still retained minor activity toward the apoptosis effector caspase-7 (Puri et al., 2012). Regardless of its off-target activity, this probe colocalized with the inflammasome adaptor protein ASC and was thus able to visualize inflammasome foci in macrophages infected with the intracellular Gram-negative pathogen S. typhimurium (Puri et al., 2012; Sanman et al., 2016a). 


\section{Imaging of glycosidase activities}

A number of ABPs have been developed to target the family of retaining glycosidases (reviewed in Kuo et al., 2018), but their application in cell-based imaging has been limited. Witte et al. developed fluorescent ABPs for glucocerebrosidase (GBA) based on the epoxide cyclophellitol, a structural analog of glucoside substrates and covalent GBA inhibitor that reacts with the nucleophilic glutamate in the active site (Witte et al., 2010). Deficiency in GBA leads to Gaucher disease, a lysosomal storage disorder. Fluorescent GBA probes specifically stained GBA in endo-lysosomal compartments in live fibroblasts and co-localized with a GBAspecific antibody (Witte et al., 2010). Biochemical analysis using the cyclophellitol probe confirmed lower GBA activities in fibroblasts from a Gaucher patient with a mutation in the $G B A$ gene compared to cells from healthy donors (Witte et al., 2010). Subsequently, Kallemeijn et al. developed probes based on a cyclophellitol-aziridine warhead which had a broader target selectivity profile compared to epoxide-based probes (Kallemeijn et al., 2012). Confocal microscopy was used to confirm lysosomal localization of the probe and its targets (Kallemeijn et al., 2012). In a recent study, glycosidase-ABPs were used to label the activity of clinically used therapeutic GBA preparations and to study their uptake into different cell types by microscopy and biochemically (Kallemeijn et al., 2017).

\section{Single-cell imaging of bacteria with antibiotic conjugates}

Antibiotics target many essential processes of bacterial cells and fluorescent antibiotic conjugates that allow the visualization of the molecular targets have emerged as versatile tools for microbial cell biology. Antibiotics interfering with the cell wall biosynthesis of bacteria can be assigned to two categories. First, penicillin and other $\beta$-lactam antibiotics target penicillin-binding proteins (PBPs) that catalyze the transglycosylation and/or transpeptidation reactions necessary to shape the peptidoglycan (or murein) sacculus during cell division. As these antibiotics covalently inhibit target enzymes, their functionalized conjugates represent bona-fide ABPs and are discussed in detail in the next section (see Böttcher and Sieber, 2012, for an early review on $\beta$-lactam and $\beta$-lactone probes). Second, substrate-binding antibiotics, such as vancomycin and ramoplanin sequester peptidoglycan (PG)-precursor substrates, preventing their proper enzymatic incorporation into the cell wall. Fluorophore-tagged analogs of both antibiotics have been developed and applied to image nascent peptidoglycan. Although they are not direct reporters of enzymatic activities and hence are not within the primary scope of this review, these probes have emerged as important tools to study the topology of PG synthesis in Bacillus subtilis and other Grampositive organisms (Daniel and Errington, 2003; Tiyanont et al., 2006).

\section{Dissecting the roles of PBPs in bacterial cell division with ABPs}

In 1999, the BOCILLIN-FL was reported as the first commercially available fluorescent penicillin derivative (Zhao et al., 1999) and it was used to detect PBPs in membrane fractions of different bacteria by SDS-PAGE analysis and fluorescence scanning (Zhao et al., 1999). Jarzembowski et al. employed BOCILLIN-FL labeling as a marker that negatively correlates with penicillin resistance in a flow cytometry study on Staphylococcus aureus and Enterococcus faecalis (Jarzembowski et al., 2008). Methicillin-resistant S. aureus (MRSA) strains had been reported to harbor subpopulations with different sensitivity to $\beta$-lactam antibiotics, and strains can be classified according to this so-called heteroresistance profile. Interestingly, flow cytometry analysis of different clinical MRSA isolates with BOCILLIN-FL uncovered different degrees of heterogeneity in probe labeling corresponding to the heteroresistance profile of the analyzed strains (Jarzembowski et al., 2009) highlighting the utility of these ABPs as molecular markers for functional states of the bacterial cells.

Kocaoglu et al. synthesized fluorescent derivatives of the $\beta$-lactam antibiotic cephalosporin C. When applied to live B. subtilis tetramethylrhodamine (TAMRA)-conjugated cephalosporin C (Ceph C-T) labeled four PBPs (assigned to $1 \mathrm{a} / 1 \mathrm{~b}, 2 \mathrm{~b}, 2 \mathrm{c}$ and 4 ), while BOCILLIN-FL additionally labeled PBPs 2a, 3 and 5 (Kocaoglu et al., 2012). Fluorescence microscopy studies revealed that Ceph C-fluorescein (Ceph C-F) labeling of cells localized to division septum, where new PG synthesis takes place (Figure 3A, Kocaoglu et al., 2012; Kocaoglu and Carlson, 2013). The authors noted that this localization was in concurrence with previous studies using green fluorescent protein (GFP)-PBP fusion proteins and immunofluorescence staining (Kocaoglu et al., 2012). Interestingly, three-dimensional (3D) super-resolution structured illumination microscopy (SIM) studies of cells co-labeled with Ceph C-T and Bocillin-FL revealed discrete localizations of PBP subpopulations corresponding to the different target specificities of the two 
A

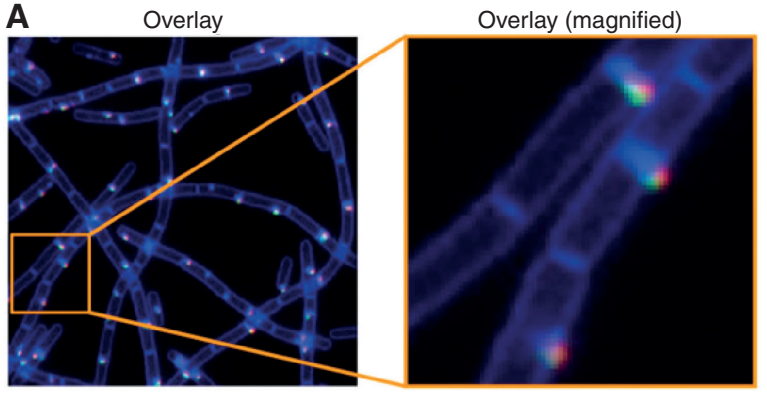

C

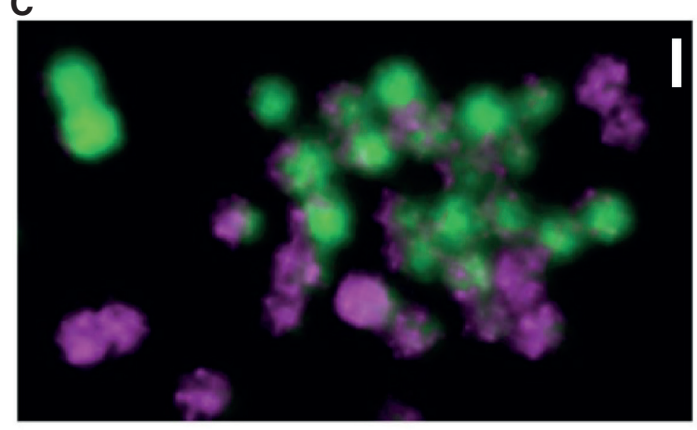

D
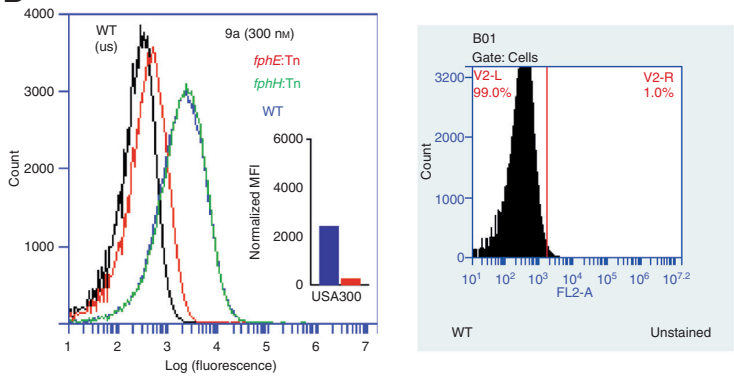

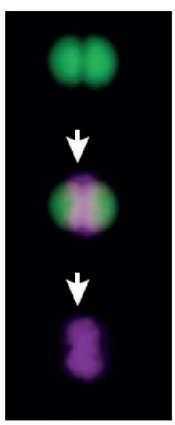

B
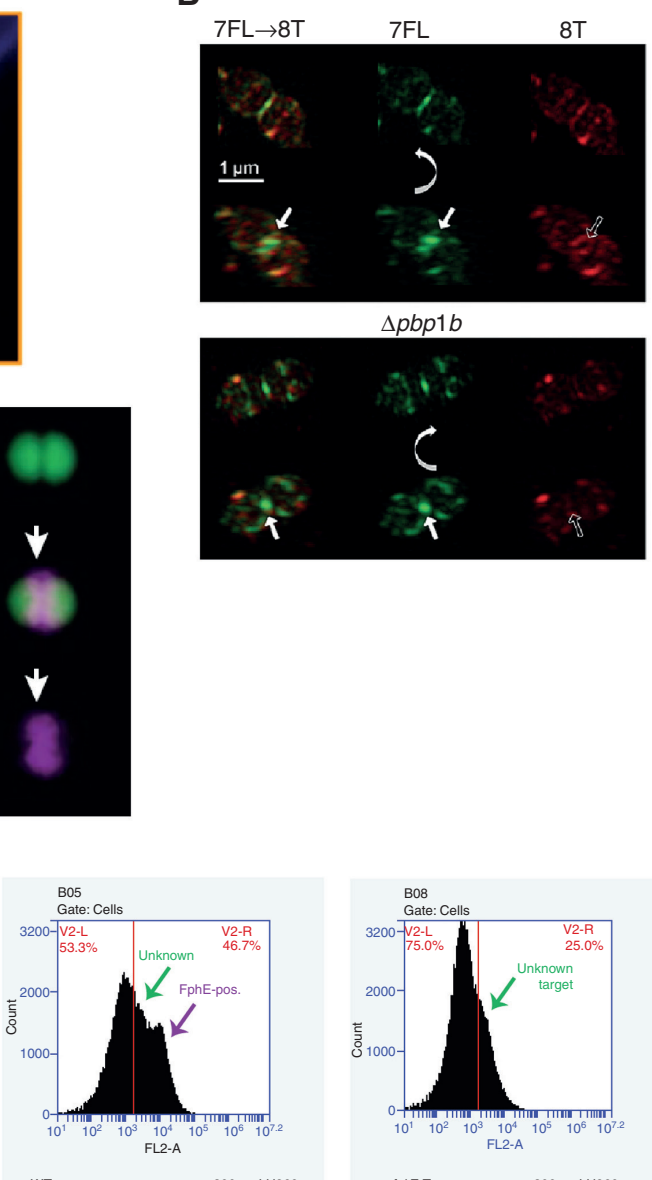

tphE:Tn

300 nм LH960

E
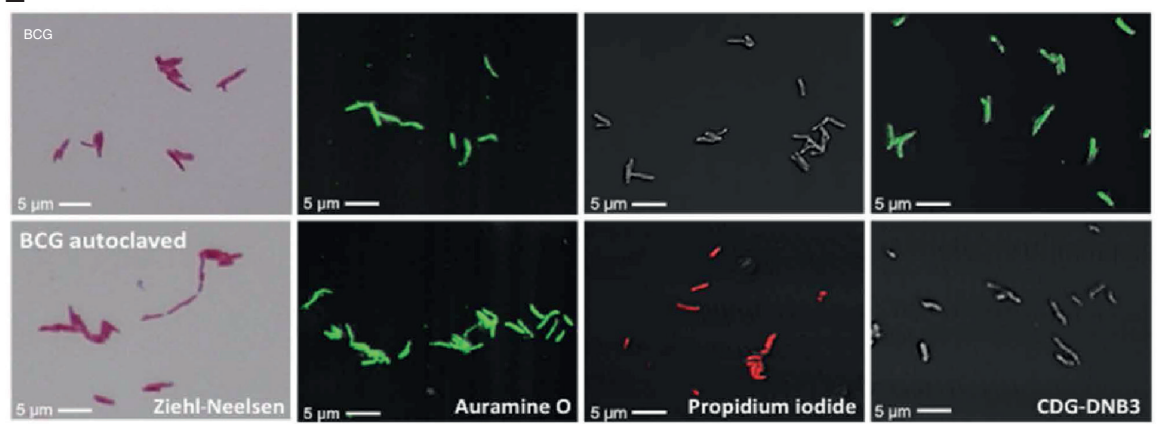

Figure 3: Examples of single-cell analysis of bacterial cells with ABPs.

(A) Wide-field microscopy mages of $B$. subtilis stained with the PBP-probes Bocillin-FL (green), cephalosporin C-T (red) and the membrane stain TMA-DPH (blue). Both probes label every other division septum where active PG synthesis occurs. The figure is reprinted with permission from Kocaoglu et al. (2012). Copyright (2012) American Chemical Society. (B) 3D-structured illumination microscopy images of dividing WT and $\Delta p b p 1 b$ S. pneumoniae labeled first with (2R,3S)- $\beta$-lactone-L-Phe-TAMRA (7FL, green) followed by (2R,3S)- $\beta$-lactone-D-PheTAMRA (8T, red). White arrows indicate septal labeling of PBP2x by 7FL, whereas PBP2b-labeling by probe $8 \mathrm{~T}$ is found peripherally and is absent from the central septum (empty arrow). The figure is reprinted with permission Sharifzadeh et al. (2017). Copyright (2017) American Chemical Society. (C) 3D reconstructions of confocal microscopy images of GFP-expressing (green) S. aureus Newman cells labeled for 30 min with the FphB-specific probe JCP251-bT (magenta). The upper graph illustrates the heterogeneity of probe labeling observed in a population of exponentially growing bacteria. The lower graph shows enrichment of JCP251-bT labeling at the cross-wall of a dividing cell during stationary phase. Scale bar: $1 \mu \mathrm{m}$. The figure were adapted from Lentz et al. (2018). (D) Flow cytometry analysis of $S$. aureus USA 300 WT, fphE-deficient or fphH-deficient labeled with $300 \mathrm{nM}$ of the fluorescent FphE-probe 9a (LH960). Probe-labeled subpopulations vary depending on growth conditions and strains used (first panel: growth on Agar plate, panels 2-4: bacteria in late stationary phase show different LH960+ subpopulations, one of which is FphE-dependent, and the other one not. The figure was adapted from Chen et al. (2019). (E) Microscopic images of freshly cultivated or autoclaved M. bovis BCG labeled by Ziehl-Neelsen staining, auramine 0, propdium iodide or the dual BlaC-DprE1-probe CDG-DNB3. The figure is reprinted with permission from Cheng et al. (2018). 
probes (Kocaoglu et al., 2012). The authors also demonstrated the utility of the Ceph C-F/Bocillin-FL labeling in the Gram-positive pathogen Streptococcus pneumoniae uncovering other distinct localization patterns of PBP subpopulations (Kocaoglu et al., 2012; Kocaoglu and Carlson, 2013). Sharifzadeh et al. later expanded the toolset of PBP imaging probes by synthesizing a small library of fluorescent $\beta$-lactone probes (Sharifzadeh et al., 2017). Although neither of the probes developed had single-target selectivity when used on their own in wild-type $S$. pneumoniae, the authors devised a dual labeling strategy (using probes 7FL and $8 \mathrm{~T}$ ) to dissect the localization pattern of $\mathrm{PBP} 2 \mathrm{~b}$ and $\mathrm{PBP} 2 \mathrm{x}$ during cell division in a $\triangle \mathrm{pbp} 1 \mathrm{~b}$ mutant strain (Pbp1 is a secondary target for both probes, Figure 3B) (Sharifzadeh et al., 2017). These studies revealed colocalization of both PBPs in early, but distinct localization patterns in mid-to-late stage division (Sharifzadeh et al., 2017).

\section{ABPs and cellular phenotypic heterogeneity in bacteria}

For human cells cellular differentiation and the existence of different cellular activation states (e.g. of immune cells) are well described. This is in part due to obvious morphological differences, but is also a result of the cluster of differentiation (CD) system of molecular markers, which allows the differentiation of cell types and functional subpopulations based on the staining of CD-surface antigens with monoclonal antibodies. For bacteria, in contrast, cellular phenotypic heterogeneity is more cryptic, although it is known that bacterial populations can organize into surface-associated spatially defined bacterial communities known as biofilms, and are capable of executing other community-associated behaviors such as division of labor and bet-hedging that involve single-cell heterogeneity (see the reviews by Ackermann, 2015; Davis and Isberg, 2016). It is also commonly appreciated that bacterial subpopulations may differ in their susceptibility toward antibacterial agents and may contain metabolically dormant antibiotic-resistant persister cells (reviewed in Harms et al., 2016). Bacterial pathogens can further modify their expressed arsenal of virulence factors depending on their growth state and microenvironment. However, the resulting functional or phenotypic differences between single cells are difficult to study and commonly require the generation of fluorescent reporter strains that monitor the activity of certain transcriptional regulators or report on the distribution of fluorescently tagged fusion protein. Our recent work on the development of ABPs for specific enzymatic targets of the opportunistic bacterial pathogen S. aureus suggests that chemical probes such as ABPs may prove highly useful for single-cell phenotypic analysis of bacterial populations and are able to distinguish functionally distinct cells by their chemical probe labeling properties (Lentz et al., 2018; Chen et al., 2019).

First, we used the $\alpha, \beta$-hydrolase probes fluorophosphonate (FP)-TMR and FP-biotin to profile serine hydrolase activities in S. aureus (Lentz et al., 2018). To our surprise, 10 out of 12 identified targets were previously uncharacterized and we decided to name them fluorophosphonate-binding serine hydrolases (Fph) A-J (Lentz et al., 2018). A competitive ABPP-screen identified a selective chloroisocoumarinbased inhibitor for $\mathrm{FphB}$, a presumed esterase and virulence factor that facilitates the infection of specific tissue sites in a mouse model of systemic infection (Lentz et al., 2018). A fluorescent analog, JCP251-bT, was confirmed as an FphBselective probe that revealed the subcellular location of its molecular target by confocal microscopy (Figure 3C). We found that JCP251-bT labeling localized to focal sites in the cell envelope and was found enriched in the septal crosswall of (some) dividing cells, the primary site of cell wall biosynthesis, suggesting a role in cell wall modification (Lentz et al., 2018). Moreover, we found a highly heterogenous labeling pattern across isogenic $S$. aureus populations, with only less than $10 \%$ of cells classified as JCP251-bT ${ }^{+}$as determined by confocal microscopy (Figure 3C) and flow cytometry (with a strict cut-off set based on background labeling assessed using an fphB-deficient strain) (Lentz et al., 2018). It is intriguing to speculate that the expression of FphB in a subset of cells under experimental conditions (liquid culture) where its activity is dispensable may generate functional diversity among the bacterial population that becomes relevant only upon encountering altered environmental conditions in certain niches (during infection).

Next, we sought to expand the chemical toolkit to study Fph activities in S. aureus and developed a 1,2,3-triazole urea-based probe whose cellular labeling profile is specific to another uncharacterized hydrolase FphE, although it also reacts with secreted $S$. aureus lipases SAL1 or SAL2 (Chen et al., 2019). Single-cell imaging and flow cytometry experiments of different $S$. aureus strains and growth environments revealed a varying composition of bacterial subpopulations classified according to the cellular FphE-probe-labeling status (Figure 3D, Chen et al., 2019). As the physiological function of FphB, FphE and other Fph hydrolases still remain under investigation, the functional relevance of the observed subpopulations currently remains unclear (Chen et al., 2019). Yet, the unique power of fluorescent ABPs to expose functional differences between isogenic cells becomes evident. 
Rao and coworkers conceived another sophisticated chemical probe labeling strategy for single-cell analysis of Mycobacterium tuberculosis (Mtb) (Xie et al., 2012; Cheng et al., 2018). With the motivation to develop Mtb-specific imaging agents for clinical Mtb diagnosis, the authors first developed a cephalosporin-based fluorogenic substrate that was specifically cleaved by the Mtb $\beta$-lactamase BlaC allowing the detection of Mtb in biological specimens (Xie et al., 2012). As diffusion of the fluorophore away from its cleavage site in the cell wall prohibits single-cell analysis and therefore also the differentiation of live or dead bacteria, the researchers conceived a dual-targeting fluorogenic probe (Cheng et al., 2018). This probe featured a caged fluorescent reporter flanked by a BlaC-substrate unit and a dinitrobenzene (DNB) warhead which is reduced by and then covalently attached to the active site cysteine of decaprenylphosphoryl- $\beta$-D-ribose 2'-epimerase (DprE1) (Cheng et al., 2018). As both BlaC and DprE1 are localized in the periplasm, $\mathrm{BlaC}$ is expected to cleave the $\beta$-lactam group, inducing a structural rearrangement that uncages the fluorophore (Cheng et al., 2018). Then the probe reacts with DprE1 thus retaining the fluorescent reporter in the cell wall. The authors demonstrate the utility of this probe to visualize Mycobacterium bovis bacille Calmette-Guérin (BCG) bacteria during macrophage infection, to differentiate live from heat-killed bacteria and to distinguish Mtb from other pathogens of the respiratory tract (Figure 3E) (Cheng et al., 2018).

\section{Interpreting chemical probe labeling - cellular factors affecting probe labeling}

It should be noted that the interpretation of any observed heterogeneity in cellular populations must take into account a number of different potential explanations, which may be attributed to 'target-related' and 'proberelated' phenomena. In the case of a hypothetic, perfectly target-selective ABP, heterogenous labeling may be attributed to individual cells or subpopulations that harbor different levels of enzymatically active target protein and can be described as 'target-related'. These differences might result from different transcription or post-translational mechanisms of enzymatic control such as limited proteolysis or allosteric activation/inhibition. It is important to note that, in reality, the reactive nature of a probe may cause its selectivity profile to change depending on the concentration of probe and the labeling times applied.
It is also possible that certain off-targets are only encountered under certain biological conditions: for example, we observed an FphE-probe ${ }^{+}$subpopulation present in S. aureus USA300 cells in stationary phase that was even present in fphE-deficient cells (Figure 3D), but was not seen in other wild-type strains or under different growth conditions (Chen et al., 2019). It is therefore highly recommended to complement imaging studies with a biochemical analysis of engaged targets.

Alternatively, cellular subpopulations may differ in the accessibility of the target to the probe ('probe-related' heterogeneity). This means that a probe-negative population may result from a lack of probe uptake or cellular permeability or be a result of efficient probe efflux. Furthermore, probes may non-specifically interact with certain cellular compartments (e.g. membrane), a behavior which can be highly dependent on the choice of fluorophore and its physico-chemical properties (Bertrand et al., 2016). Importantly, 'target-related heterogeneity' can be regarded as 'target-specific', whereas 'probe-related heterogeneity' can be expected to be a less specific phenomenon that would apply to a broader variety of xenobiotics of a similar size and charge.

Thus, simple dyes can already become useful chemical tools in probing cellular heterogeneity resulting from altered uptake or efflux. Kumar et al., for example, used the fluorescent mitochondrial dye MitoTrackerGreen to stain tissue-resident memory $\mathrm{T}$ cells revealing subpopulations of Mito ${ }^{\text {hi }}$ and Mito ${ }^{\text {lo }}$ cells (Kumar et al., 2017). The authors demonstrated that Mito $^{\text {lo }}$ cells result from dye efflux by applying competitive inhibitors of efflux pumps leading to loss of the Mito ${ }^{\text {lo }}$ population and uniform cellular staining as Mito ${ }^{\text {hi }}$ population (Kumar et al., 2017).

A prime example of both the utility and the complexity of chemical probe labeling for single-cell analysis is the recent work by Rego and colleagues who used the non-specific fluorogenic esterase substrate calcein-acetoxymethyl (AM) to microscopically image single-cell heterogeneity in Mycobacterium smegmatis (Rego et al., 2017). The authors reasoned that the fluorescence signal, which across individual bacteria varied over two orders of magnitude as a function of uptake, esterase activity and efflux, would be a proxy for cellular accessibility to xenobiotics in general (Rego et al., 2017). Indeed, bright cells showed reduced likelihood of survival after treatment with the antibiotic rifampicin compared to darker cells that may have taken up less dye and less antibiotic (Rego et al., 2017). In a flow cytometry assay that uses the distribution of the calcein-AM fluorescence signal in the M. smegmatis cellular population as a readout, the authors then identified loss of asymmetry mutant A (lamA) in a transposon mutant 
screen (Rego et al., 2017). This new mycobacterial divisome factor asymmetrically inhibits cell wall synthesis during the transition of cell division to elongation, generating single-cell heterogeneity and promoting antibiotic resistance of M. tuberculosis (Rego et al., 2017).

\section{Beyond imaging - activity-based probes in single-cell chemical proteomics}

While the previous examples illustrate the power of ABPs for spatio-temporal imaging or as functional markers for cellular phenotypes, they only provide a one-dimensional readout characterized as the intensity of probe labeling for each cell. In order to provide a second dimension of information on the different targets engaged by a probe in a single cell, Liu and coworkers developed a single-cell chemical proteomics system (Xu et al., 2014; Chen et al., 2016). In this device, droplets encapsulating single cells are injected through a microfluidic injector into a capillary electrophoresis-laser induced fluorescence (CE-LIF) detection system (Xu et al., 2014; Chen et al., 2016). Inside the capillary, cells are lysed by SDS treatment and heating before subjected to CE-LIF analysis, where fluorescently labeled proteins are detected after separation by molecular weight as displayed in an electropherogram (Xu et al., 2014; Chen et al., 2016). In a first study, a tri-modular probe was designed to target the GB1 subunit of the gammaaminobutyric acid $\mathrm{B}\left(\mathrm{GABA}_{\mathrm{B}}\right)$ receptor (Xu et al., 2014). The probe was based on a reversibly binding antagonist scaffold, it featured a photoactivatable crosslinker (diazirine) to irreversibly attach the probe to its molecular target upon ultraviolet (UV) irradiation, and it possessed a fluorophore for detection (Xu et al., 2014). Using this setup the authors were able to detect quantitative differences in GB1 levels in different neurons and also different protein peak sizes, likely corresponding to different splice variants or posttranslational modifications prevalent in individual cells (Xu et al., 2014). In a second report, the CE-LIF technology was used to analyze the composition of cathepsins in single cells of HeLa and RAW264.7 cell lines using an epoxysuccinyl-based probe. In the electropherograms two distinct peaks were resolved corresponding to families of cathepsin with similar molecular weight (Cat H, L1, S, F, O at $\sim 26.6 \mathrm{kDa}$ and Cat B,Z at $\sim 30 \mathrm{kDa}$ ), while in RAW cells an additional peak at $\sim 50 \mathrm{kDa}$ was present (Chen et al., 2016). Intriguingly, ABP-labeling patterns of single cells clustered together in subpopulations of cells with similar cysteine protease activity patterns, i.e. electropherograms of individual RAW cells either featured the $26 \mathrm{kDa}$ and $30 \mathrm{kDa}$ peak, or the $30 \mathrm{kDa}$ and $50 \mathrm{kDa}$ peak (Chen et al., 2016).

\section{Outlook}

The use of fluorescent ABPs has enlightened many aspects of mammalian and prokaryotic cell biology and has contributed to our understanding of enzymatic processes in a cellular context. ABPs may find utility as analytical markers for certain cellular fates, e.g. in cell death or immune cell activation. In other instances, the phenotypically distinct subpopulations defined by ABP labeling in single-cell studies are less well understood: it is unclear why individual cells are different from each other, to what extent they are different (i.e. how they differ beyond their ability to stain differently with ABPs and corresponding differences in either target activity and/or accessibility) and how this relates to the physiological role of molecular target of the $\mathrm{ABP}$. In order to uncover the new biology underlying these questions, ABPs hold another promise: they can be used as functional markers allowing for the purification of phenotypically distinct cellular subpopulations by fluorescenceactivated cell sorting (FACS), thus enabling their separate downstream analysis, e.g. by 'omics' technologies or in functional assays. In contrast to other fluorescent markers used in biological studies that require the generation of genetically modified reporter strains, chemical probes such as ABPs can be easily applied to a variety of different native biological systems. Their use as phenotypic markers in single-cell studies should therefore find broad use in different areas of biology and medicine.

Acknowledgments: The author thanks Brett Babin and Mark Brönstrup for helpful suggestions and proof-reading of the manuscript.

\section{References}

Abd-Elrahman, I., Meir, K., Kosuge, H., Ben-Nun, Y., Weiss Sadan, T., Rubinstein, C., Samet, Y., McConnell, M.V., and Blum, G. (2016). Characterizing cathepsin activity and macrophage subtypes in excised human carotid plaques. Stroke 47, 1101-1108.

Ackermann, M. (2015). A functional perspective on phenotypic heterogeneity in microorganisms. Nat. Rev. Microbiol. 13, 497-508.

Bedner, E., Smolewski, P., Amstad, P., and Darzynkiewicz, Z. (2000). Activation of caspases measured in situ by binding of fluorochrome-labeled inhibitors of caspases (FLICA): correlation with DNA fragmentation. Exp. Cell Res. 259, 308-313. 
Berger, A.B., Vitorino, P.M., and Bogyo, M. (2004). Activity-based protein profiling: applications to biomarker discovery, in vivo imaging and drug discovery. Am. J. Pharmacogenomics 4, 371-381.

Bertrand, R., Wolf, A., Ivashchenko, Y., Lohn, M., Schafer, M., Bronstrup, M., Gotthardt, M., Derdau, V., and Plettenburg, O. (2016). Synthesis and characterization of a promising novel FFAR1/ GPR40 targeting fluorescent probe for $\beta$-cell imaging. ACS Chem. Biol. 11, 1745-1754.

Blum, G., Mullins, S.R., Keren, K., Fonovic, M., Jedeszko, C., Rice, M.J., Sloane, B.F., and Bogyo, M. (2005). Dynamic imaging of protease activity with fluorescently quenched activity-based probes. Nat. Chem. Biol. 1, 203-209.

Blum, G., von Degenfeld, G., Merchant, M.J., Blau, H.M., and Bogyo, M. (2007). Noninvasive optical imaging of cysteine protease activity using fluorescently quenched activity-based probes. Nat. Chem. Biol. 3, 668-677.

Borne, A.L., Huang, T., McCloud, R.L., Pachaiyappan, B., Bullock, T.N.J., and Hsu, K.L. (2019). Deciphering T cell immunometabolism with activity-based protein profiling. Curr. Top Microbiol. Immunol. 420, 175-210.

Böttcher, T. and Sieber, S.A. (2012). $\beta$-Lactams and $\beta$-lactones as activity-based probes in chemical biology. MedChemComm. 3, 408-417.

Chakrabarty, S., Kahler, J.P., van de Plassche, M.A.T., Vanhoutte, R., and Verhelst, S.H.L. (2019). Recent advances in activity-based protein profiling of proteases. Curr. Top Microbiol. Immunol. 420, 253-281.

Chen, D., Fan, F., Zhao, X., Xu, F., Chen, P., Wang, J., Ban, L., Liu, Z., Feng, X., Zhang, Y., et al. (2016). Single cell chemical proteomics with membrane-permeable activity-based probe for identification of functional proteins in lysosome of tumors. Anal. Chem. 88, 2466-2471.

Chen, L., Keller, L.J., Cordasco, E., Bogyo, M., and Lentz, C.S. (2019). Fluorescent triazole urea activity-based probes for the singlecell phenotypic characterization of Staphylococcus aureus. Angew. Chem. Int. Ed. 58, 5643-5647.

Cheng, Y., Xie, J., Lee, K.H., Gaur, R.L., Song, A., Dai, T., Ren, H., Wu, J., Sun, Z., Banaei, N., et al. (2018). Rapid and specific labeling of single live Mycobacterium tuberculosis with a dual-targeting fluorogenic probe. Sci. Transl. Med. 10, pii: eaar4470.

Daniel, R.A. and Errington, J. (2003). Control of cell morphogenesis in bacteria: two distinct ways to make a rod-shaped cell. Cell $113,767-776$.

Davis, K.M. and Isberg, R.R. (2016). Defining heterogeneity within bacterial populations via single cell approaches. Bioessays 38 , 782-790.

Desrochers, G.F. and Pezacki, J.P. (2019). ABPP and host-virus interactions. Curr. Top Microbiol. Immunol. 420, 131-154.

Edgington, L.E., Berger, A.B., Blum, G., Albrow, V.E., Paulick, M.G., Lineberry, N., and Bogyo, M. (2009). Noninvasive optical imaging of apoptosis by caspase-targeted activity-based probes. Nat. Med. 15, 967-973.

Edgington, L.E., van Raam, B.J., Verdoes, M., Wierschem, C., Salvesen, G.S., and Bogyo, M. (2012). An optimized activitybased probe for the study of caspase- 6 activation. Chem. Biol. 19, 340-352.

Edgington-Mitchell, L.E., Wartmann, T., Fleming, A.K., Gocheva, V., van der Linden, W.A., Withana, N.P., Verdoes, M., Aurelio, L., Edgington-Mitchell, D., Lieu, T., et al. (2016). Legumain is activated in macrophages during pancreatitis. Am. J. Physiol. Gastrointest. Liver Physiol. 311, G548-560.

Garland, M., Yim, J.J., and Bogyo, M. (2016). A bright future for precision medicine: advances in fluorescent chemical probe design and their clinical application. Cell Chem. Biol. 23, 122-136.

Gocheva, V., Wang, H.W., Gadea, B.B., Shree, T., Hunter, K.E., Garfall, A.L., Berman, T., and Joyce, J.A. (2010). IL-4 induces cathepsin protease activity in tumor-associated macrophages to promote cancer growth and invasion. Genes Dev. 24, 241-255.

Guerra, M., Frey, D., Hagner, M., Dittrich, S., Paulsen, M., Mall, M.A., and Schultz, C. (2019). Cathepsin G activity as a new marker for detecting airway inflammation by microscopy and flow cytometry. ACS Cent. Sci. 5, 539-548.

Harms, A., Maisonneuve, E., and Gerdes, K. (2016). Mechanisms of bacterial persistence during stress and antibiotic exposure. Science 354, pii: aaf4268.

Harris, J.L., Backes, B.J., Leonetti, F., Mahrus, S., Ellman, J.A., and Craik, C.S. (2000). Rapid and general profiling of protease specificity by using combinatorial fluorogenic substrate libraries. Proc. Natl. Acad. Sci. USA 97, 7754-7759.

Hu, H.Y., Gehrig, S., Reither, G., Subramanian, D., Mall, M.A., Plettenburg, O., and Schultz, C. (2014). FRET-based and other fluorescent proteinase probes. Biotechnol. J. 9, 266-281.

Jarzembowski, T., Wisniewska, K., Jozwik, A., Bryl, E., and Witkowski, J. (2008). Flow cytometry as a rapid test for detection of penicillin resistance directly in bacterial cells in Enterococcus faecalis and Staphylococcus aureus. Curr. Microbiol. 57, 167-169.

Jarzembowski, T., Wisniewska, K., Jozwik, A., and Witkowski, J. (2009). Heterogeneity of methicillin-resistant Staphylococcus aureus strains (MRSA) characterized by flow cytometry. Curr. Microbiol. 59, 78-80.

Joyce, J.A., Baruch, A., Chehade, K., Meyer-Morse, N., Giraudo, E., Tsai, F.Y., Greenbaum, D.C., Hager, J.H., Bogyo, M., and Hanahan, D. (2004). Cathepsin cysteine proteases are effectors of invasive growth and angiogenesis during multistage tumorigenesis. Cancer Cell 5, 443-453.

Kallemeijn, W.W., Li, K.Y., Witte, M.D., Marques, A.R., Aten, J., Scheij, S., Jiang, J., Willems, L.I., Voorn-Brouwer, T.M., van Roomen, C.P., et al. (2012). Novel activity-based probes for broadspectrum profiling of retaining $\beta$-exoglucosidases in situ and in vivo. Angew. Chem. Int. Ed. 51, 12529-12533.

Kallemeijn, W.W., Scheij, S., Hoogendoorn, S., Witte, M.D., Herrera Moro Chao, D., van Roomen, C.P., Ottenhoff, R., Overkleeft, H.S., Boot, R.G., and Aerts, J.M. (2017). Investigations on therapeutic glucocerebrosidases through paired detection with fluorescent activity-based probes. PLoS One 12, e0170268.

Kasperkiewicz, P., Poreba, M., Snipas, S.J., Parker, H., Winterbourn, C.C., Salvesen, G.S., and Drag, M. (2014). Design of ultrasensitive probes for human neutrophil elastase through hybrid combinatorial substrate library profiling. Proc. Natl. Acad. Sci. USA 111, 2518-2523.

Kasperkiewicz, P., Poreba, M., Snipas, S.J., Lin, S.J., Kirchhofer, D., Salvesen, G.S., and Drag, M. (2015). Design of a selective substrate and activity based probe for human neutrophil serine protease 4. PLoS One 10, e0132818.

Kasperkiewicz, P., Altman, Y., D’Angelo, M., Salvesen, G.S., and Drag, M. (2017). Toolbox of fluorescent probes for parallel imaging reveals uneven location of serine proteases in neutrophils. J. Am. Chem. Soc. 139, 10115-10125. 
Kato, D., Boatright, K.M., Berger, A.B., Nazif, T., Blum, G., Ryan, C., Chehade, K.A., Salvesen, G.S., and Bogyo, M. (2005). Activitybased probes that target diverse cysteine protease families. Nat. Chem. Biol. 1, 33-38.

Kocaoglu, O. and Carlson, E.E. (2013). Penicillin-binding protein imaging probes. Curr. Protoc. Chem. Biol. 5, 239-250.

Kocaoglu, O., Calvo, R.A., Sham, L.T., Cozy, L.M., Lanning, B.R., Francis, S., Winkler, M.E., Kearns, D.B., and Carlson, E.E. (2012). Selective penicillin-binding protein imaging probes reveal substructure in bacterial cell division. ACS Chem. Biol. 7, 1746-1753.

Koenders, S.T.A., Gagestein, B., and van der Stelt, M. (2019). Opportunities for lipid-based probes in the field of immunology. Curr. Top Microbiol. Immunol. 420, 283-319.

Kovalyova, Y. and Hatzios, S.K. (2019). Activity-based protein profiling at the host-pathogen interface. Curr. Top Microbiol. Immunol. 420, 73-91.

Kumar, B.V., Ma, W., Miron, M., Granot, T., Guyer, R.S., Carpenter, D.J., Senda, T., Sun, X., Ho, S.H., Lerner, H., et al. (2017). Human tissue-resident memory $T$ cells are defined by core transcriptional and functional signatures in lymphoid and mucosal sites. Cell Rep. 20, 2921-2934.

Kuo, C.L., van Meel, E., Kytidou, K., Kallemeijn, W.W., Witte, M., Overkleeft, H.S., Artola, M.E., and Aerts, J.M. (2018). Activitybased probes for glycosidases: profiling and other applications. Methods Enzymol. 598, 217-235.

Lentz, C.S., Ordonez, A.A., Kasperkiewicz, P., La Greca, F., O’Donoghue, A.J., Schulze, C.J., Powers, J.C., Craik, C.S., Drag, M., Jain, S.K., et al. (2016). Design of selective substrates and activity-based probes for hydrolase important for pathogenesis 1 (HIP1) from Mycobacterium tuberculosis. ACS Infect. Dis. 2, 807-815.

Lentz, C.S., Sheldon, J.R., Crawford, L.A., Cooper, R., Garland, M., Amieva, M.R., Weerapana, E., Skaar, E.P., and Bogyo, M. (2018). Identification of a $S$. aureus virulence factor by activitybased protein profiling (ABPP). Nat. Chem. Biol. 14, 609-617.

Liu, Y., Patricelli, M.P., and Cravatt, B.F. (1999). Activity-based protein profiling: the serine hydrolases. Proc. Natl. Acad. Sci. USA 96, 14694-14699.

Liu, S.Y., Xiong, H., Li, R.R., Yang, W.C., and Yang, G.F. (2019). Activity-based near-infrared fluorogenic probe enables in vitro and in vivo profiling of neutrophil elastase. Anal. Chem. 91, 3877-3884.

Man, S.M., Karki, R., and Kanneganti, T.D. (2017). Molecular mechanisms and functions of pyroptosis, inflammatory caspases and inflammasomes in infectious diseases. Immunol. Rev. 277, 61-75.

Morimoto, K. and van der Hoorn, R.A. (2016). The increasing impact of activity-based protein profiling in plant science. Plant Cell Physiol. 57, 446-461.

Niphakis, M.J. and Cravatt, B.F. (2014). Enzyme inhibitor discovery by activity-based protein profiling. Annu. Rev. Biochem. 83, 341-377.

Nomura, D.K. and Maimone, T.J. (2019). Target identification of bioactive covalently acting natural products. Curr. Top Microbiol. Immunol. 420, 351-374.

Nomura, D.K., Dix, M.M., and Cravatt, B.F. (2010). Activity-based protein profiling for biochemical pathway discovery in cancer. Nat. Rev. Cancer 10, 630-638.

O’Donoghue, A.J., Eroy-Reveles, A.A., Knudsen, G.M., Ingram, J., Zhou, M., Statnekov, J.B., Greninger, A.L., Hostetter, D.R.,
Qu, G., Maltby, D.A., et al. (2012). Global identification of peptidase specificity by multiplex substrate profiling. Nat. Methods 9, 1095-1100.

Ofori, L.O., Withana, N.P., Prestwood, T.R., Verdoes, M., Brady, J.J., Winslow, M.M., Sorger, J., and Bogyo, M. (2015). Design of protease activated optical contrast agents that exploit a latent lysosomotropic effect for use in fluorescence-guided surgery. ACS Chem. Biol. 10, 1977-1988.

Patel, H.V., Li, M., and Seeliger, J.C. (2019). Opportunities and challenges in activity-based protein profiling of mycobacteria. Curr. Top Microbiol. Immunol. 420, 49-72.

Patterson, A.W., Wood, W.J., Hornsby, M., Lesley, S., Spraggon, G., and Ellman, J.A. (2006). Identification of selective, nonpeptidic nitrile inhibitors of cathepsin S using the substrate activity screening method. J. Med. Chem. 49, 6298-6307.

Poreba, M., Kasperkiewicz, P., Snipas, S.J., Fasci, D., Salvesen, G.S., and Drag, M. (2014). Unnatural amino acids increase sensitivity and provide for the design of highly selective caspase substrates. Cell Death Differ. 21, 1482-1492.

Poreba, M., Rut, W., Vizovisek, M., Groborz, K., Kasperkiewicz, P., Finlay, D., Vuori, K., Turk, D., Turk, B., Salvesen, G.S., et al. (2018). Selective imaging of cathepsin L in breast cancer by fluorescent activity-based probes. Chem. Sci. 9, 2113-2129.

Pozarowski, P., Huang, X., Halicka, D.H., Lee, B., Johnson, G., and Darzynkiewicz, Z. (2003). Interactions of fluorochrome-labeled caspase inhibitors with apoptotic cells: a caution in data interpretation. Cytometry A 55, 50-60.

Puri, A.W., Broz, P., Shen, A., Monack, D.M., and Bogyo, M. (2012). Caspase-1 activity is required to bypass macrophage apoptosis upon Salmonella infection. Nat. Chem. Biol. 8, 745-747.

Rego, E.H., Audette, R.E., and Rubin, E.J. (2017). Deletion of a mycobacterial divisome factor collapses single-cell phenotypic heterogeneity. Nature 546, 153-157.

Roth-Konforti, M.E., Bauer, C.R., and Shabat, D. (2017). Unprecedented sensitivity in a probe for monitoring cathepsin B: chemiluminescence microscopy cell-imaging of a natively expressed enzyme. Angew. Chem. Int. Ed. 56, 15633-15638.

Sanman, L.E. and Bogyo, M. (2014). Activity-based profiling of proteases. Annu. Rev. Biochem. 83, 249-273.

Sanman, L.E., Qian, Y., Eisele, N.A., Ng, T.M., van der Linden, W.A., Monack, D.M., Weerapana, E., and Bogyo, M. (2016a). Disruption of glycolytic flux is a signal for inflammasome signaling and pyroptotic cell death. eLife 5, e13663.

Sanman, L.E., van der Linden, W.A., Verdoes, M., and Bogyo, M. (2016b). Bifunctional probes of cathepsin protease activity and ph reveal alterations in endolysosomal $\mathrm{pH}$ during bacterial infection. Cell Chem. Biol. 23, 793-804.

Sharifzadeh, S., Boersma, M.J., Kocaoglu, O., Shokri, A., Brown, C.L., Shirley, J.D., Winkler, M.E., and Carlson, E.E. (2017). Novel electrophilic scaffold for imaging of essential penicillin-binding proteins in Streptococcus pneumoniae. ACS Chem. Biol. 12, 2849-2857.

Sharifzadeh, S., Shirley, J.D., and Carlson, E.E. (2019). Activitybased protein profiling methods to study bacteria: the power of small-molecule electrophiles. Curr. Top Microbiol. Immunol. 420, 23-48.

Shaulov-Rotem, Y., Merquiol, E., Weiss-Sadan, T., Moshel, O., Salpeter, S., Shabat, D., Kaschani, F., Kaiser, M., and Blum, G. (2016). A novel quenched fluorescent activity-based probe reveals caspase- 3 activity in the endoplasmic reticulum during apoptosis. Chem. Sci. 7, 1322-1337. 
Siegrist, M.S., Swarts, B.M., Fox, D.M., Lim, S.A., and Bertozzi, C.R. (2015). Illumination of growth, division and secretion by metabolic labeling of the bacterial cell surface. FEMS Microbiol. Rev. 39, 184-202.

Tiyanont, K., Doan, T., Lazarus, M.B., Fang, X., Rudner, D.Z., and Walker, S. (2006). Imaging peptidoglycan biosynthesis in Bacillus subtilis with fluorescent antibiotics. Proc. Natl. Acad. Sci. USA 103, 11033-11038.

Turk, V., Stoka, V., Vasiljeva, O., Renko, M., Sun, T., Turk, B., and Turk, D. (2012). Cysteine cathepsin S: from structure, function and regulation to new frontiers. Biochim. Biophys. Acta. 1824, 68-88.

van Esbroeck, A.C.M., Janssen, A.P.A., Cognetta, A.B., 3rd, Ogasawara, D., Shpak, G., van der Kroeg, M., Kantae, V., Baggelaar, M.P., de Vrij, F.M.S., Deng, H., et al. (2017). Activity-based protein profiling reveals off-target proteins of the FAAH inhibitor BIA 10-2474. Science 356, 1084-1087.

van Kasteren, S.I., Florea, B.I., and Overkleeft, H.S. (2017). Activitybased protein profiling: from chemical novelty to biomedical stalwart. Methods Mol. Biol. 1491, 1-8.

Verdoes, M., Edgington, L.E., Scheeren, F.A., Leyva, M., Blum, G., Weiskopf, K., Bachmann, M.H., Ellman, J.A., and Bogyo, M. (2012). A nonpeptidic cathepsin S activity-based probe for noninvasive optical imaging of tumor-associated macrophages. Chem. Biol. 19, 619-628.

Verdoes, M., Oresic Bender, K., Segal, E., van der Linden, W.A., Syed, S., Withana, N.P., Sanman, L.E., and Bogyo, M. (2013). Improved quenched fluorescent probe for imaging of cysteine cathepsin activity. J. Am. Chem. Soc. 135, 14726-14730.

Vocadlo, D.J. and Bertozzi, C.R. (2004). A strategy for functional proteomic analysis of glycosidase activity from cell lysates. Angew. Chem. Int. Ed. 43, 5338-5342.

Whidbey, C. and Wright, A.T. (2019). Activity-based protein profilingenabling multimodal functional studies of microbial communities. Curr. Top Microbiol. Immunol. 420, 1-21.

Witte, M.D., Kallemeijn, W.W., Aten, J., Li, K.Y., Strijland, A., DonkerKoopman, W.E., van den Nieuwendijk, A.M., Bleijlevens, B., Kramer, G., Florea, B.I., et al. (2010). Ultrasensitive in situ visualization of active glucocerebrosidase molecules. Nat. Chem. Biol. 6, 907-913.
Wright, M.H. and Sieber, S.A. (2016). Chemical proteomics approaches for identifying the cellular targets of natural products. Nat. Prod. Rep. 33, 681-708.

Xie, H., Mire, J., Kong, Y., Chang, M., Hassounah, H.A., Thornton, C.N., Sacchettini, J.C., Cirillo, J.D., and Rao, J. (2012). Rapid point-of-care detection of the tuberculosis pathogen using a BlaC-specific fluorogenic probe. Nat. Chem. 4, 802-809.

Xu, F., Zhao, H., Feng, X., Chen, L., Chen, D., Zhang, Y., Nan, F., Liu, J., and Liu, B.F. (2014). Single-cell chemical proteomics with an activity-based probe: identification of low-copy membrane proteins on primary neurons. Angew. Chem. Int. Ed. 53, 6730-6733.

Zhao, G., Meier, T.I., Kahl, S.D., Gee, K.R., and Blaszczak, L.C. (1999). BOCILLIN FL, a sensitive and commercially available reagent for detection of penicillin-binding proteins. Antimicrob. Agents Chemother. 43, 1124-1128.

\section{Bionote}

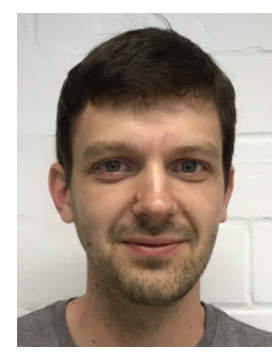

\section{Christian S. Lentz}

Department of Chemical Biology (CBIO), Helmholtz-Centre for Infection Research, Inhoffenstr. 7, D-38102 Braunschweig, Germany christian.lentz@helmholtz-hzi.de. https://orcid.org/0000-0001-7284-2264

Christian Lentz studied Molecular Biomedicine at the University of Bonn, Germany, where he also obtained his PhD on the identification of heme biosynthesis inhibitors of endosymbiotic bacteria in parasitic worms. During his postdoctoral work in the lab of Matthew Bogyo at Stanford University, California, he became intrigued about the versatility of activity-based probes and their application for molecular imaging. His current research at the Helmholtz-Center for Infection Research in Braunschweig, Germany, focuses on the dissection of functional diversity of bacterial pathogens using activity-based probes. 